\title{
Thrombus Permeability on Dynamic CTA Predicts Good Outcome after Reperfusion Therapy
}

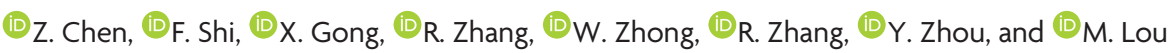

\section{ABSTRACT}

BACKGROUND AND PURPOSE: Thrombus permeability assessed on conventional CTA is associated with neurologic outcome in patients with acute ischemic stroke. We aimed to investigate whether dynamic CTA can improve the accuracy of thrombus permeability assessment and its predictive value for outcome.

MATERIALS AND METHODS: We reviewed consecutive patients with acute ischemic stroke who had occlusion of the Ml segment of the middle artery cerebral artery and underwent pretreatment perfusion $\mathrm{CT}$. Thrombus permeability, determined by thrombus attenuation increase (TAI), was assessed on 26-phase dynamic CTA derived from perfusion CT. TAI ${ }_{\text {max }}$ was defined as the maximum TAI among phases; $\mathrm{TAl}_{\text {peak, }}$ as TAl of peak arterial phase; $\mathrm{TAl}_{\text {con, }}$, as TAl on phase 13. Good outcome was defined as a 3-month mRS score of $\leq 2$.

RESULTS: One hundred four patients were enrolled in the final analysis. The median TAI $\mathrm{max}_{\text {, }} \mathrm{TAI}_{\text {peak }}$, and TAI $\mathrm{I}_{\text {con }}$ were $30.1 \mathrm{HU}$ (interquartile range, $13.0-50.2 \mathrm{HU}$ ), $9.5 \mathrm{HU}$ (interquartile range, $-1.6-28.7 \mathrm{HU}$ ), and $6.6 \mathrm{HU}$ (interquartile range, $-5.1-24.4 \mathrm{HU}$ ), respectively. Multivariable regression analyses showed that $\mathrm{TAI}_{\max }(\mathrm{OR}=1.027 ; 95 \% \mathrm{Cl}, 1.007-1.048 ; P=.008), \mathrm{TAl}_{\text {peak }}(\mathrm{OR}=1.029 ; 95 \% \mathrm{Cl}, 1.005-1.054 ; P=.020)$, and $\mathrm{TAI}_{\text {con }}(\mathrm{OR}=1.026 ; 95 \% \mathrm{Cl}, 1.002-1.051 ; P=.037)$ were independently associated with good outcome. The areas under the ROC curve of $\mathrm{TAl}_{\text {max }}, \mathrm{TAl}_{\text {peak }}$, and $\mathrm{TAl}_{\text {con }}$ in predicting good outcome were $0.734,0.701$, and 0.658 , respectively.

CONCLUSIONS: Thrombus permeability assessed on dynamic CTA could be a better predictor of outcome after reperfusion therapy than that assessed on conventional single-phase CTA.

ABBREVIATIONS: AIS = acute ischemic stroke; AUC = area under the curve; IQR = interquartile range; $\mathrm{PH}=$ parenchymal hemorrhage; ONT = onset to intravenous thrombolysis; $\mathrm{ROC}=$ receiver operating characteristic; $\mathrm{TAI}=$ thrombus attenuation increase; $\mathrm{TAl} \mathrm{I}_{\text {con }}=\mathrm{TAl}$ on phase $13 ; \mathrm{TAl}$ max $=$ the maximum TAl among phases; TAl $_{\text {peak }}=$ TAl of peak arterial phase

C erebral large-artery occlusion accounts for about one-third of acute ischemic strokes (AISs), which may cause severe disability and high mortality rates. ${ }^{1-6}$ Effort has been made on neuroimaging to predict the outcome of acute ischemic stroke be-

Received May 17, 2018; accepted after revision July 17

From the Department of Neurology (Z.C., F.S., X.G., R.Z., W.Z., R.Z., Y.Z., M.L.), Second Affiliated Hospital of Zhejiang University, School of Medicine, Hangzhou, China; and Zhejiang University Brain Research Institute (M.L.), Hangzhou, Zhejiang, China.

Z. Chen and F. Shi contributed equally to this work.

This study was supported by the National Key Research and Development Program of China (2016YFC1301500), the National Natural Science Foundation of China (81622017, 81471170, and 81601017), Fundamental Research Funds for the Central Universities (2017XZZX002-09), and Zhejiang Provincial Natural Science

Foundation (LQ16H090003)

Please address correspondence to Min Lou, MD, PhD, Department of Neurology, the Second Affiliated Hospital of Zhejiang University, School of Medicine, No. 88 Jiefang Rd, Hangzhou, China, 310009; e-mail: loumingxc@vip.sina.com

-- Indicates open access to non-subscribers at www.ajnr.org

三 Indicates article with supplemental on-line table.

http://dx.doi.org/10.3174/ajnr.A5785 cause improved neurologic outcome is the goal for treatment. Thrombus characteristics on admission imaging, such as clot length, density, and location, may have the potential to predict the outcome of patients with AIS under different treatments. ${ }^{7}$

In patients with AIS, the thrombus is commonly regarded as a cork where the artery is completely occluded. ${ }^{8}$ The permeability of thrombi has been well-characterized in preclinical studies. ${ }^{9-11}$ It has been suggested that thrombus permeability might be related to the physical porosity of thrombus and might reflect the ability of soluble molecules to move within the gaps among adjacent platelets, fibrin filaments, and red blood cells. ${ }^{8,9,12,13}$ Preclinical studies have demonstrated that high permeability of the thrombus within the occluded artery allowed residual blood to flow through the thrombus, ${ }^{9,10}$ which may have a positive effect on neurologic outcome after acute ischemia due to the compensating oxygenation of brain tissue distal to the occluded artery. Recently, thrombus attenuation increase (TAI) was used to assess the thrombus permeability on conventional single-phase CT angiography and noncontrast CT. A pervious thrombus with a high TAI 
was found to be associated with better neurologic outcome after reperfusion therapy in patients with AIS. ${ }^{8,12}$

The enhancement of arteries on conventional single-phase CTA is affected by the acquisition time because the contrast filling of the occlusion site is delayed compared with that in the normal condition. ${ }^{14}$ Therefore, the permeability of the thrombi may be underestimated if single-phase CTA is acquired before contrast completely penetrates the thrombus. Compared with singlephase CTA, dynamically acquired CTA provides a broad temporal coverage that spans from unenhanced through to the arterial and, subsequently, venous phases. In addition, previous studies have demonstrated that dynamic CTA can better characterize the intracranial thrombus burden than single-phase CTA. ${ }^{14}$

We thus hypothesized that dynamic CTA could overcome the timing limitation and improve the assessment accuracy of thrombus permeability. We then assessed thrombus permeability on 26-phase dynamic CTA derived from CT perfusion and aimed to investigate its predictive value for neurologic outcome in patients with AIS after reperfusion therapy.

\section{MATERIALS AND METHODS \\ Patient Inclusion}

We retrospectively reviewed our prospectively collected data base for consecutive patients with AIS who received intravenous thrombolysis with or without endovascular thrombectomy from May 2009 to February 2017. Then, we enrolled patients who fulfilled the following criteria: 1) They had a diagnosis of AIS confirmed by diffusion-weighted imaging or CT at 24 hours after symptom onset, 2) underwent CTP within 8 hours after stroke onset, 3) had occlusion of the M1 segment of the middle cerebral artery without involvement of the internal carotid artery, 4) underwent CTA or time-of-flight MR angiography at 24 hours after treatment, and 5) underwent follow-up NCCT or susceptibilityweighted imaging at 24 hours after treatment. We excluded patients who had poor image quality due to motion artifacts or incomplete consecutive acquisitions.

\section{Ethics Statement}

Ethical approval was obtained from the human ethics committee of our center. The clinical investigation was conducted according to the principles expressed in the Declaration of Helsinki. Written informed consent was obtained from all patients.

\section{Imaging Parameters}

CTP was performed on a 64-slice CT scanner (Somatom Definition Flash; Siemens, Erlangen, Germany), including an NCCT scan $(120 \mathrm{kV}, 320 \mathrm{~mA}$, contiguous 5-mm axial slices, 7-second acquisition time) and volume CTP (100 $\mathrm{mm}$ in the $\mathrm{z}$-axis, 4 -second delay after the start of contrast material bolus injection, 74.5second total imaging duration, $80 \mathrm{kV}, 120 \mathrm{~mA}, 1.5-\mathrm{mm}$ slice thickness, $32 \times 1.2 \mathrm{~mm}$ collimation). Volume CTP consisted of 26 consecutive spiral acquisitions of the brain. All 26 scans were divided into 4 parts: 1) two scans with 3 -second cycle time, 2) fifteen scans with 1.5-second cycle time, 3 ) four scans with 3-second cycle time, and 4) five scans with 6-second cycle time. Axial slice coverage was $150 \mathrm{~mm}$. A $60-\mathrm{mL}$ bolus of contrast medium (iopamidol, Imeron; Bracco Sine, Shanghai, China) with a single injection was used at a flow rate of $6 \mathrm{~mL} / \mathrm{s}$, followed by a $20-\mathrm{mL}$ saline chaser at $6 \mathrm{~mL} / \mathrm{s}$.

\section{Imaging Analysis}

The Arterial Occlusive Lesion Scale was used to assess recanalization on 24-hour MRA or CTA (grade 0: complete occlusion of the target artery; grade 1: incomplete occlusion or partial local recanalization at the target artery with no distal flow; grade 2: incomplete occlusion or partial local recanalization at the target artery with any distal flow; grade 3: complete recanalization and restoration of the target artery with any distal flow). ${ }^{15}$ Recanalization and no recanalization were defined as arterial occlusive lesion grades $2-3$ and $0-1$, respectively. Hemorrhagic transformation was assessed on 24-hour SWI or NCCT according to the second European-Australasian Acute Stroke Study (ECASS II), including hemorrhagic infarction and parenchymal hemorrhage $(\mathrm{PH}){ }^{16}$ Previously validated thresholds were applied to measure the baseline hypoperfusion volume (time-to-maximum $>6$ seconds) ${ }^{17}$ and infarct core volume (relative cerebral blood flow of $<30 \%$ ) on CTP. ${ }^{18}$

\section{Thrombus Permeability Assessment}

Thrombus permeability assessments were conducted on commercial software (MIStar; Apollo Medical Imaging Technology, Melbourne, Australia). The 26-phase dynamic CTA with 4.5$\mathrm{mm}$-thick maximum intensity projection was reconstructed from CTP source images. The proximal artery of the contralateral hemisphere was selected to generate the arterial input function curve. The measurements of TAI were performed as described previously. $8,12,19$ Three ROIs with a radius of $1 \mathrm{~mm}$ were placed on the thrombus by 2 experienced neuroradiologists blinded to the patients' information, with rater discrepancies settled by consensus discussion; the mean attenuation on each phase was calculated. The ROIs might partially overlap each other in case of a small thrombus. The mean attenuation of the thrombus on phase 1 was set as the reference value. The TAI of phase 2 to phase 26 was defined as the increase of the mean attenuation of the thrombus from phase 1 to each phase, respectively. Three parameters of TAI, including $\mathrm{TAI}_{\text {max }}$, $\mathrm{TAI}_{\text {peak }}$, and $\mathrm{TAI}_{\text {con }}$, were used for the final analyses (Fig 1). TAI max $_{\text {ax }}$ was defined as the maximal TAI among 25 phases (phases 2-26). TAI peak was defined as the TAI of the arterial peak phase according to the arterial input function curve. The mean time between contrast injection and peak concentration on the normal side of the middle cerebral artery was $25 \mathrm{sec}$ onds (phase 13) in current study; thus, TAI $_{\text {con }}$ was simulated as TAI on phase 13 of dynamic CTA.

\section{Clinical Data}

We reviewed demographic, clinical, and radiologic data, including age; sex; prior antiplatelet use; risk factors such as smoking, hypertension, diabetes mellitus, hyperlipidemia, smoking, and atrial fibrillation; time interval from stroke onset to intravenous thrombolysis (ONT); National Institutes of Health Stroke Scale score on admission; baseline hypoperfusion volume and infarct core volume on admission; hemorrhagic transformation and recanalization after intravenous thrombolysis; and modified Rankin Scale score after 3 months. Patients were dichotomized 


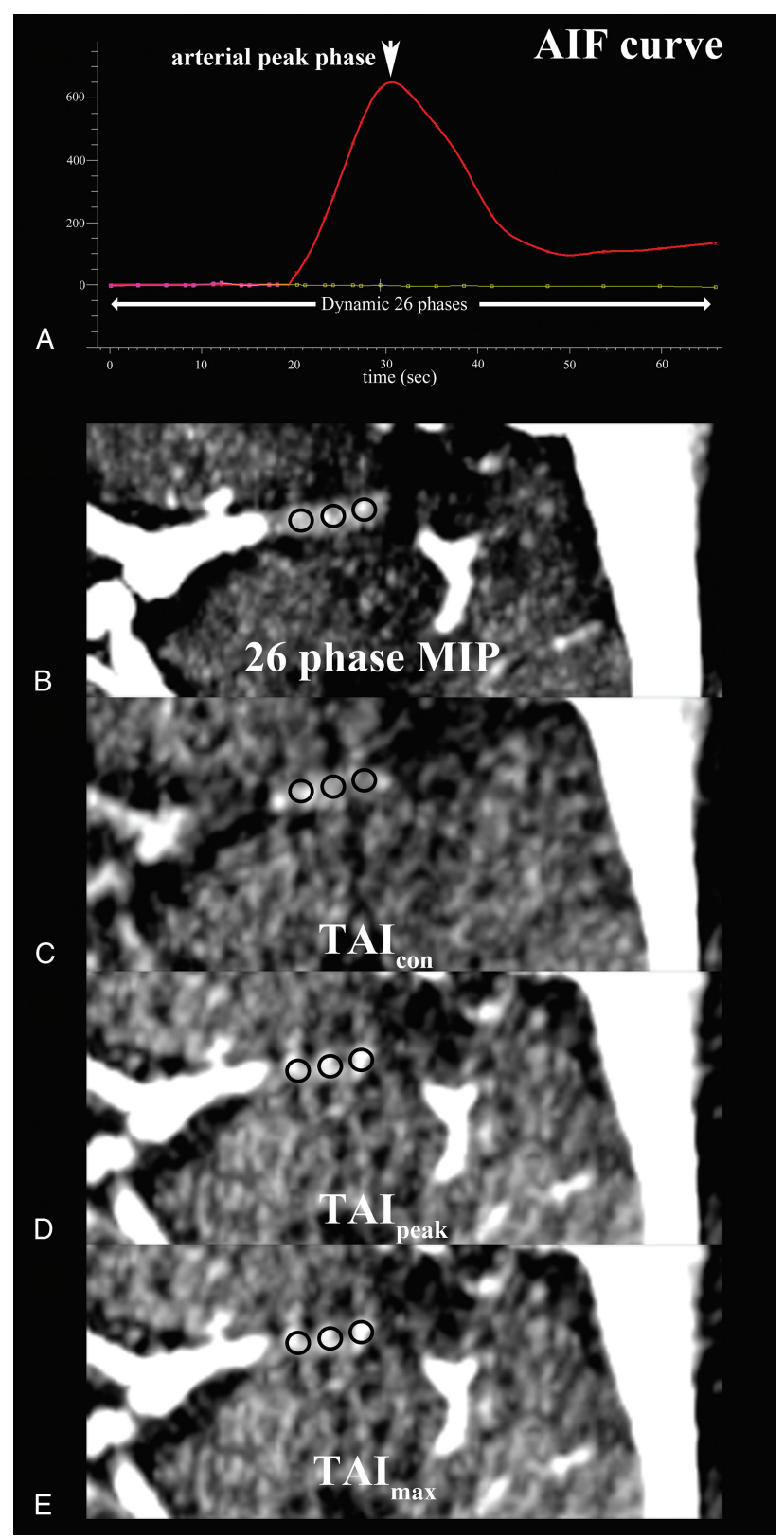

FIG 1. Illustration of thrombus attenuation increase assessment with 3 ROIs (black circles) measuring the average thrombus attenuations on a left $\mathrm{Ml}$ segment of the middle cerebral artery on motion-corrected dynamic CTA. The arterial input function (AIF) curve for all 26 phases was generated $(A)$ and the thrombus permeability was assessed on the CTA images with maximum intensity projection (MIP) in all 26 phases $(B)$, at the mean time delay following intravenous contrast injection in the current study (phase 13) (C), at the arterial peak phase (phase 18) (D), and at the phase with the maximum value (phase 22) (E) of the mean attenuation increase.

into good ( $\mathrm{mRS}$ score of $\leq 2$ ) and poor outcome (mRS score of $>2$ ) at 90 days.

\section{Statistical Analysis}

Mean with SD, medians with interquartile range (IQR), and percentages were used to describe the distribution of continuous and categoric variables. The Fisher exact test was used to compare categoric variables among groups, whereas the independent-samples 2-tailed $t$ test or the Mann-Whitney $U$ test was used for the continuous variables, as appropriate. The Spearman rank correlation test was used to test the association of TAI with clinical and imaging variables. The strength of these associations was compared using the area under the receiver operating characteristic (ROC) curve. The ROC-derived optimal cutoff was determined at the maximal Youden Index. Variables with a $P$ value $<.1$ in univariate analyses were enrolled in the multivariable regression model. A $P$ value $<.05$ was considered statistically significant. All statistical analyses were performed with SPSS, Version 22.0 (IBM, Armonk, New York).

\section{RESULTS}

\section{Overall Characteristics}

One hundred four patients with AIS with MCA-M1 were enrolled in the final analysis. The mean age was $68 \pm 14$ years, the mean NIHSS score was $14 \pm 6$ on admission, and 61 (58.7\%) patients were male. The median ONT was 215 (IQR, 140-295) minutes. The median $\mathrm{TAI}_{\text {max }}, \mathrm{TAI}_{\text {peak }}$ and $\mathrm{TAI}_{\text {con }}$ were $30.1 \mathrm{HU}$ (IQR, 13.0-50.2 HU), 9.5 HU (IQR, - 1.6-28.7 HU), and 6.6 HU (IQR, $-5.1-24.4 \mathrm{HU})$, respectively.

\section{Association of TAI with Radiologic and Neurologic Outcome}

As shown in Table 1, 44 (42.3\%) patients achieved good outcome. Patients with good outcome had higher $\mathrm{TAI}_{\max }, \mathrm{TAI}_{\text {peak }}$, and $\mathrm{TAI}_{\text {con }}$ than those with poor outcome $(P<.001, P<.001$, and $P=.006$, respectively). Patients with good outcome were younger $(P=.005)$ and had a lower baseline NIHSS score $(P<.001)$, lower baseline infarct core volume $(P=.001)$, lower baseline hypoperfusion volume $(P=.004)$, and higher recanalization rates (84\% versus $64 \%, P=.023$ ) compared with those with poor outcome.

After we adjusted for age, baseline NIHSS score, atrial fibrillation, ONT, baseline infarct core volume, $\mathrm{TAI}_{\max }(\mathrm{OR}=1.027$; 95\% CI, 1.007-1.048; $P=.008), \mathrm{TAI}_{\text {peak }}(\mathrm{OR}=1.029 ; 95 \% \mathrm{CI}$, $1.005-1.054 ; P=.020)$, and $\mathrm{TAI}_{\text {con }}(\mathrm{OR}=1.026$; $95 \% \mathrm{CI}, 1.002-$ $1.051 ; P=.037)$ were independently associated with good outcome, respectively. Patients with $\mathrm{PH}$ had lower $\mathrm{TAI}_{\max }$ and $\mathrm{TAI}_{\text {peak }}$ than those without $\mathrm{PH}$, while TAI $\mathrm{I}_{\text {con }}$ did not show a significant difference between patients with and without $\mathrm{PH}(P=$ $.308)$.

The ROC curves of $\mathrm{TAI}_{\text {max }}, \mathrm{TAI}_{\text {peak }}$, and $\mathrm{TAI}_{\text {con }}$ in predicting good outcome are shown in Fig 2, and the areas under the curve (AUCs) were $0.734,0.701$, and 0.658 , respectively. The AUCs of each single-phase CTA derived from CTP are shown in On-line Table. The optimal cutoffs were 30.9, 9.0, and 14.6 HU for $\mathrm{TAI}_{\text {max }}, \mathrm{TAI}_{\text {peak }}$ and $\mathrm{TAI}_{\text {con }}$, respectively. Patients were then dichotomized into a pervious thrombus group and an impervious thrombus group according to the optimal cutoff values of TAI ${ }_{\text {max }}$, $\mathrm{TAI}_{\text {peak, }}$, and $\mathrm{TAI}_{\text {con }}$, respectively. After we adjusted for age, baseline NIHSS score, atrial fibrillation, ONT, and baseline infarct core volume, patients with a pervious thrombus had a higher rate of good outcome than those with an impervious thrombus when

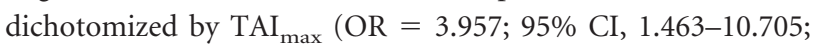
$P=.007)$ and $\mathrm{TAI}_{\text {peak }}(\mathrm{OR}=2.887 ; 95 \% \mathrm{CI}, 1.075-7.754 ; P=$ .035) (Table 2). However, when dichotomized by $\mathrm{TAI}_{\text {con }}$, patients with a pervious thrombus were not independently associated with 


\begin{tabular}{|c|c|c|c|}
\hline & $\begin{array}{c}\text { Poor Outcome } \\
(m R S>2)(n=60)\end{array}$ & $\begin{array}{l}\text { Good Outcome } \\
(m R S \leq 2)(n=44)\end{array}$ & $P$ Value \\
\hline Age (yr) & $74(65-81)$ & $66(58-76)$ & .005 \\
\hline Male (No.) (\%) & $33(55.0 \%)$ & $28(63.6 \%)$ & .424 \\
\hline Baseline NIHSS (mean) & $16 \pm 5$ & $11 \pm 6$ & $<.001$ \\
\hline Onset-to-needle time (mean) (min) & 230 (168-297) & 207 (119-259) & .059 \\
\hline Endovascular thrombectomy (No.) (\%) & $22(36.7 \%)$ & $16(36.4 \%)$ & 1.000 \\
\hline Prior antiplatelet usage (No.) (\%) & $12(20.0 \%)$ & $9(20.5 \%)$ & 1.000 \\
\hline \multicolumn{4}{|l|}{ Risk factors } \\
\hline Smoking (No.) (\%) & $18(30.0 \%)$ & $13(29.5 \%)$ & 1.000 \\
\hline Hypertension (No.) (\%) & $35(58.3 \%)$ & $22(50.0 \%)$ & .431 \\
\hline Diabetes mellitus (No.) (\%) & $16(26.7 \%)$ & $6(13.6 \%)$ & .146 \\
\hline Hyperlipidemia (No.) (\%) & $22(36.7 \%)$ & $21(47.7 \%)$ & .315 \\
\hline History of stroke/TIA (No.) (\%) & $11(18.3 \%)$ & $5(11.4 \%)$ & .415 \\
\hline Atrial fibrillation (No.) (\%) & $38(63.3 \%)$ & $20(45.5 \%)$ & .076 \\
\hline \multicolumn{4}{|l|}{ Radiologic data } \\
\hline Baseline infarct core volume (mean) (mL) & $69.27 \pm 49.51$ & $40.72 \pm 30.91$ & .001 \\
\hline Baseline hypoperfusion volume (mean) (mL) & $134.28 \pm 61.07$ & $98.22 \pm 60.43$ & .004 \\
\hline $\mathrm{TAI}_{\max }$ (median) (IQR) (HU) & $18.0(10.0-38.7)$ & $39.9(26.1-73.3)$ & $<.001$ \\
\hline $\mathrm{TAI}_{\text {peak }}$ (median) (IQR) (HU) & $4.6(-4.2-17.5)$ & $18.7(5.9-43.0)$ & $<.001$ \\
\hline $\mathrm{TAI}_{\text {con }}$ (median) (IQR) (HU) & $2.2(-5.5-12.4)$ & $10.4(-2.0-36.7)$ & .006 \\
\hline Thrombus perviousness by dichotomized TAI max (No.) (\%) & $18(30.0 \%)$ & $31(70.5 \%)$ & $<.001$ \\
\hline Thrombus perviousness by dichotomized TAI peak (No.) (\%) & $22(36.7 \%)$ & $31(70.5 \%)$ & .001 \\
\hline Thrombus perviousness by dichotomized TAI con (No.) (\%) & $13(21.7 \%)$ & $21(47.7 \%)$ & .005 \\
\hline
\end{tabular}

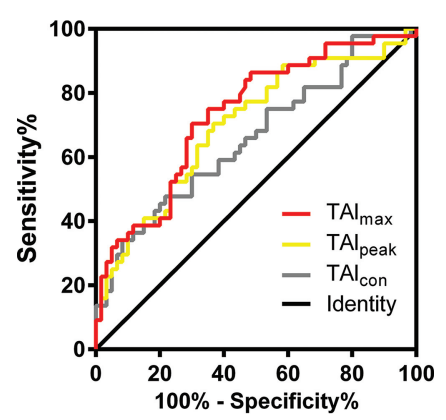

FIG 2. ROC curve of thrombus attenuation increase for $T A I_{\max }$, $\mathrm{TAI}_{\text {peak }}$, and $\mathrm{TAI}_{\text {con }}$, respectively.

good outcome $(\mathrm{OR}=2.323 ; 95 \% \mathrm{CI}, 0.815-6.621 ; P=.115)$ (Table 2).

\section{DISCUSSION}

In the present study, we found that high thrombus permeability on dynamic CTA is an independent predictor of good outcome in patients with AIS. Most interesting, TAI $_{\max }$ and TAI $\mathrm{T}_{\text {peak }}$ derived from CTP data are better imaging markers than $\mathrm{TAI}_{\text {con }}$ for predicting neurologic outcome.

Our finding that the permeable thrombus was strongly associated with good outcome is consistent with previous studies using conventional single-phase CTA. ${ }^{8,12}$ These studies also reported that the association between permeable thrombi and good outcome was independent of recanalization status. ${ }^{8}$ High thrombus permeability promotes anterograde filling of blood, and, therefore, less severely ischemic brain tissue.

Most important, our study revealed that the AUCs of TAI $\mathrm{max}_{\max }$ and $\mathrm{TAI}_{\text {peak }}$ for predicting good outcome $(0.734$ and 0.701$)$ were higher than those of $\mathrm{TAI}_{\mathrm{con}}(\mathrm{AUC}=0.658)$ and previously reported values (AUC $=0.67$ ) using conventional single-phase CTA; these findings indicate that thrombus permeability assessed on dynamic CTA might be a better biomarker for predicting out-
Table 2: Multivariable regression for good outcome

\begin{tabular}{|c|c|c|c|}
\hline & OR & $95 \% \mathrm{Cl}$ & $\begin{array}{c}P \\
\text { Value }\end{array}$ \\
\hline \multicolumn{4}{|l|}{ Model 1} \\
\hline Age & 0.966 & $0.926-1.008$ & .114 \\
\hline Baseline NIHSS & 0.911 & $0.823-1.008$ & .071 \\
\hline Atrial fibrillation & 0.872 & $0.298-2.555$ & .803 \\
\hline ONT & 0.992 & $0.987-0.997$ & .004 \\
\hline Baseline infarct core volume & 0.986 & $0.972-1.001$ & .061 \\
\hline $\begin{array}{l}\text { Thrombus perviousness by } \\
\text { dichotomized } \mathrm{TAI}_{\max }\end{array}$ & 3.957 & $1.463-10.705$ & .007 \\
\hline \multicolumn{4}{|l|}{ Model 2} \\
\hline Age & 0.963 & $0.924-1.004$ & .074 \\
\hline Baseline NIHSS & 0.912 & $0.826-1.006$ & .066 \\
\hline Atrial fibrillation & 0.736 & $0.260-2.082$ & .564 \\
\hline ONT & 0.992 & $0.987-0.997$ & .003 \\
\hline Baseline infarct core volume & 0.988 & $0.974-1.002$ & .093 \\
\hline $\begin{array}{l}\text { Thrombus perviousness by } \\
\text { dichotomized } \mathrm{TAl}_{\text {peak }}\end{array}$ & 2.887 & $1.075-7.754$ & .035 \\
\hline \multicolumn{4}{|l|}{ Model 3} \\
\hline Age & 0.965 & $0.926-1.004$ & .080 \\
\hline Baseline NIHSS & 0.917 & $0.827-1.016$ & .098 \\
\hline Atrial fibrillation & 0.751 & $0.269-2.102$ & .586 \\
\hline ONT & 0.992 & $0.987-0.997$ & .003 \\
\hline Baseline infarct core volume & 0.986 & $0.972-1.000$ & .050 \\
\hline $\begin{array}{l}\text { Thrombus perviousness by } \\
\text { dichotomized TAI }_{\text {con }}\end{array}$ & 2.323 & $0.815-6.621$ & .115 \\
\hline
\end{tabular}

come. Usually, single-phase CTA is used to assess thrombus permeability by calculating the attenuation increase before and after contrast penetrating the thrombus. The thrombus permeability on single-phase CTA might be underestimated due to timing limitations, hemodynamic restriction, or even pseudo-occlusion. ${ }^{14,20}$ It was reported that delayed phases after the arterial peak phase on dynamic CTA could provide better thrombus depiction and prognostic information and thus might affect treatment decisions in the acute setting. ${ }^{14,21}$ A previous study using 3-phase CTA found that arterial phase CTA was superior to venous phase CTA (8 seconds after arterial phase CTA) or delayed-phase CTA 
(16 seconds after arterial phase CTA) to assess the TAI. ${ }^{22}$ However, 3-phase CTA did not cover enough time points, and the optimal phase could be located between the arterial phase and venous phase. Thus, we believe dynamic CTA derived from CTP was better for evaluating clot perviousness. Moreover, the characteristics of the arterial input function curve were affected by cardiac function, vascular curvature, and vascular stenosis. Thus, parameters based on individual hemodynamic characteristics derived from CTP data can better represent thrombus permeability than conventional single-phase CTA. Furthermore, the evaluation of dynamic CTA has no additional $\mathrm{x}$-ray exposure and contrast medium usage because it is based on CTP, which can also provide information about infarct core and penumbra.

To the best of our knowledge, we are the first to have found that low thrombus permeability was associated with a high rate of $\mathrm{PH}$ after reperfusion therapy. It has been shown that compensating oxygenation to ischemic brain tissue could reduce hemorrhagic transformation after thrombolysis. ${ }^{23}$ Therefore, we postulate that permeable thrombi may enhance tissue oxygenation distal to the occlusion due to increased passage of blood, which reduces the rate of $\mathrm{PH}$. Additionally, the negative correlation between TAI and baseline NIHSS score, infarct core volume, and hypoperfusion volume might imply less severity of ischemia in patients with pervious thrombi, which could be related to lower rates of $\mathrm{PH}$.

We did not find a significant association between thrombus permeability and recanalization, inconsistent with previous studies, ${ }^{8,12}$ which may be due to the inclusion of patients who received endovascular thrombectomy because endovascular thrombectomy usually has higher recanalization with mechanical retrieval devices. Another reason may be due to the assessment of recanalization at 24 hours after stroke in our study, while previous studies underwent follow-up imaging at 3 days or even later. ${ }^{8,12}$ Besides, the sample size of the current study was relatively small, and all patients were from a single center. Thus, further investigations in larger and multicenter cohorts are needed.

This study has several limitations. First, this was a retrospective design and might have a potential risk of selection bias, though the data were prospectively collected using the same stroke registry and CTP protocol. Second, although the ROI placement was performed by 2 experts blinded to the patients' information with rater discrepancies settled by consensus discussion, variation still exists within a thrombus. Therefore, the mean attenuation of the 3 ROIs selected may not represent the entire thrombus. Third, the overlapping vessels, calcified clots, and vascular calcification might influence the attenuation measurements, though the ROIs were placed very carefully to avoid them. Fourth, further research about blood flow status on DSA is needed to explain the association between thrombus perviousness and good outcome. Finally, the sample size was modest, and all the samples were from a single center. Confirmation and extension of these findings in larger and multicenter cohorts are needed.

\section{CONCLUSIONS}

Our study suggests that thrombus permeability assessed on dynamic CTA could be a better predictor of outcome after reperfu- sion therapy than that assessed on conventional single-phase CTA.

Disclosures: Zhicai Chen—RELATED: Grant: National Natural Science Foundation of China (81601017) and Zhejiang Provincial Natural Science Foundation of China (LQ16H090003).* Min Lou—RELATED: Grant: National Natural Science Foundation of China (81622017 and 81471170), Comments: National Natural Science Foundation of China (81622017 and 81471170), the National Key Research and Development Program of China (2016 YFC1301500), and Fundamental Research Funds for the Central Universities (2017XZZX002-09).* * Money paid to the institution.

\section{REFERENCES}

1. Gorelick PB, Wong KS, Bae HJ, et al. Large artery intracranial occlusive disease: a large worldwide burden but a relatively neglected frontier. Stroke 2008;39:2396-99 CrossRef Medline

2. Saver JL, Jahan R, Levy EI, et al. Solitaire flow restoration device versus the Merci Retriever in patients with acute ischaemic stroke (SWIFT): a randomised, parallel-group, non-inferiority trial. Lancet 2012;380:1241-49 CrossRef Medline

3. van Seeters T, Biessels GJ, Kappelle LJ, et al; Dutch Acute Stroke Study (DUST) investigators. The prognostic value of CT angiography and CT perfusion in acute ischemic stroke. Cerebrovasc Dis 2015;40: 258-69 CrossRef Medline

4. Malhotra K, Gornbein J, Saver JL. Ischemic strokes due to largevessel occlusions contribute disproportionately to stroke-related dependence and death: a review. Front Neurol 2017;8:651 CrossRef Medline

5. Lima FO, Silva GS, Furie KL, et al. Field assessment stroke triage for emergency destination: a simple and accurate prehospital scale to detect large vessel occlusion strokes. Stroke 2016;47: 1997-2002 CrossRef Medline

6. Beumer D, Mulder MJ, Saiedie G, et al. Occurrence of intracranial large vessel occlusion in consecutive, non-referred patients with acute ischemic stroke. Neurovascular Imaging 2016;2:11-975 CrossRef

7. Yoo AJ, Pulli B, Gonzalez RG. Imaging-based treatment selection for intravenous and intra-arterial stroke therapies: a comprehensive review. Expert Rev Cardiovasc Ther 2011;9:857-76 CrossRef Medline

8. Santos EM, Marquering HA, den Blanken MD, et al; MR CLEAN Investigators. Thrombus permeability is associated with improved functional outcome and recanalization in patients with ischemic stroke. Stroke 2016;47:732-41 CrossRef Medline

9. Voronov RS, Stalker TJ, Brass LF, et al. Simulation of intrathrombus fluid and solute transport using in vivo clot structures with single platelet resolution. Ann Biomed Eng 2013;41:1297-307 CrossRef Medline

10. Laurens N, Koolwijk P, de Maat MP. Fibrin structure and wound healing. J Thromb Haemost 2006;4:932-39 CrossRef Medline

11. Brass LF, Wannemacher KM, Ma P, et al. Regulating thrombus growth and stability to achieve an optimal response to injury. $J$ Thromb Haemost 2011;9(Suppl 1):66-75 CrossRef Medline

12. Santos EM, Dankbaar JW, Treurniet KM, et al; DUST Investigators. Permeable thrombi are associated with higher intravenous recombinant tissue-type plasminogen activator treatment success in patients with acute ischemic stroke. Stroke 2016;47:2058-65 CrossRef Medline

13. Welsh JD, Stalker TJ, Voronov R, et al. A systems approach to hemostasis: 1 . The interdependence of thrombus architecture and agonist movements in the gaps between platelets. Blood 2014;124: 1808-15 CrossRef Medline

14. Frölich AM, Schrader D, Klotz E, et al. 4D CT angiography more closely defines intracranial thrombus burden than single-phase CT angiography. AJNR Am J Neuroradiol 2013;34:1908-13 CrossRef Medline

15. Khatri P, Neff J, Broderick JP, et al; IMS-I Investigators. Revascularization end points in stroke interventional trials: recanalization versus reperfusion in IMS-I. Stroke 2005;36:2400-03 CrossRef Medline 
16. Hacke W, Kaste M, Fieschi C, et al. Randomised double-blind placebo-controlled trial of thrombolytic therapy with intravenous alteplase in acute ischaemic stroke (ECASS II). Lancet 1998;352: 1245-51 CrossRef

17. Lin L, Bivard A, Levi CR, et al. Comparison of computed tomographic and magnetic resonance perfusion measurements in acute ischemic stroke: back-to-back quantitative analysis. Stroke 2014;45: 1727-32 CrossRef Medline

18. Campbell BC, Christensen S, Levi CR, et al. Cerebral blood flow is the optimal CT perfusion parameter for assessing infarct core. Stroke 2011;42:3435-40 CrossRef Medline

19. Santos EM, Yoo AJ, Beenen LF, et al; MR CLEAN investigators. Observer variability of absolute and relative thrombus density measurements in patients with acute ischemic stroke. Neuroradiology 2016;58:133-39 CrossRef Medline
20. Marquering HA, Nederkoorn PJ, Beenen LF, et al. Carotid pseudoocclusion on CTA in patients with acute ischemic stroke: a concerning observation. Clin Neurol Neurosurg 2013;115:1591-94 CrossRef Medline

21. Byrne D, Sugrue G, Stanley E, et al. Improved detection of anterior circulation occlusions: the "delayed vessel sign" on multiphase CT angiography. AJNR Am J Neuroradiol 2017;38:1911-16 CrossRef Medline

22. Santos EM, d'Esterre CD, Treurniet KM, et al; PRove-IT investigators. Added value of multiphase CTA imaging for thrombus perviousness assessment. Neuroradiology 2018;60:71-79 CrossRef Medline

23. Sun L, Zhou W, Mueller C, et al. Oxygen therapy reduces secondary hemorrhage after thrombolysis in thromboembolic cerebral ischemia. J Cereb Blood Flow Metab 2010;30:1651-60 CrossRef Medline 